\title{
e-Phaïstos
}

e-Phaïstos

Revue d'histoire des techniques / Journal of the history

of technology

IV-2 | 2015

Patrimoines de l'eau

\section{Le béton armé dans le concept de patrimoine à préserver}

Reinforced concrete in the concept of heritage to be preserved

\section{Mariangela Licordari}

\section{(2) OpenEdition}

Journals

Édition électronique

URL : http://journals.openedition.org/ephaistos/792

DOI : 10.4000/ephaistos.792

ISSN : 2552-0741

Éditeur

IHMC - Institut d'histoire moderne et contemporaine (UMR 8066)

Édition imprimée

Date de publication : 1 octobre 2015

Pagination : 72-82

ISSN : 2262-7340

Référence électronique

Mariangela Licordari, « Le béton armé dans le concept de patrimoine à préserver », e-Phaïstos [En ligne], IV-2 | 2015, mis en ligne le 29 novembre 2016, consulté le 20 avril 2019. URL : http:// journals.openedition.org/ephaistos/792 ; DOI : 10.4000/ephaistos.792 


\section{Le béton armé dans le concept de patrimoine à préserver}

\begin{abstract}
«...quand nous construisons, pensons que nous sommes en train de construire pour toujours. Et ne le faisons pas pour notre satisfaction d'aujourd'hui, ni pour la seule utilité du moment; que notre ouvrage soit tel que nos descendants nous rendront grâce. ${ }^{1}$
\end{abstract}

Chaque élément naturel, chaque ensemble de structures créées par l'homme dans un lieu et un temps donnés, ainsi que ses modifications anthropiques ultérieures, génèrent une identité environnementale unique et spécifique, liée à une unicité qui vient de la parfaite intégration entre urbanisme, architecture et nature, qui est à la base de la distinction d'un endroit à l'autre, d'un panorama à l'autre. Le paysage dans ce sens, bien dynamique et complexe, est formé par des facteurs naturels en constante évolution auxquels l'homme se rapporte pour définir sa propre image sur le territoire et sur l'environnement. Les résultats, plus ou moins satisfaisants selon la capacité intellectuelle et la sensibilité du cas, conduisent à une nouvelle perception de l'ensemble qui, dans les exemples les plus riches, se trouve en parfaite harmonie avec l'environnement alentour.

Dans ces cas, l'expérience humaine qui dérive est celle de la perception et de la jouissance de la beauté, qui s'incarne dans la réalité physique qui la

\section{Mariangela Licordari}

Master Erasmus Mundus TPTI, promotion 5 « Cinque Terre », Université Paris I - Panthéon Sorbonne (France), Université d'Evora (Portugal) et Université de Padoue (Italie). véhicule en se présentant sur la scène mondiale avec sa propre physionomie, avec une histoire, avec des origines données, et aussi avec la capacité d'offrir des informations sur l'espace et le temps dans lesquels elle a été conçue, et de la façon dont elle a été perçue, dans un renouvellement continu de soi-même. Il faut, en effet, se rappeler que la beauté qui se concrétise dans un objet réel et physique, à savoir une œuvre d'art (dans ce cas architectonique ou d'ingénierie), enferme en soimême tant les qualités artistiques et techniques de l'époque dans laquelle elle a été conçue, que les aspirations et les attentes de son auteur, ou bien encore des qualités complémentaires. On entend par cela ce que la même œuvre a acquis avec l'écoulement du temps, avec l'évolution de la société et la sensibilité du regard avec lequel l'œuvre même est perçue, dans une mutation continue de soimême. Avec cette idée, à mon avis, on comprend le concept de bien culturel. C'est à travers cette présence physique de la beauté, qui donc devient plus tangible et plus compréhensible quand la beauté est liée à un bien matériel, qu'une res bien définie (architecture, peinture, statue, etc.) devient bien culturel. À cela s'ajoute la notion de mémoire et de témoignage historique que le bien possède, qui ne doit jamais être oubliée. 
Si on revient au concept de paysage, alors, la relation intrinsèque entre l'homme et la nature, qui est à la base de l'identification d'un lieu, devient aussi intéressante, surtout si elle est entendue comme un moyen par lequel on peut avoir une connaissance réelle et physique de la beauté de ce lieu. Par conséquent, un bâtiment comme un pont, une route, un aqueduc ou une centrale hydroélectrique doivent être considérés et perçus en tant qu'instruments de connaissance de cet espace ; à savoir, en tant qu'instrument qui entre dans le paysage pour en devenir un «indicateur » spécifique. À celui-ci est ensuite ajouté la particularité principale d'une construction basée sur un projet, c'est-à-dire sa capacité de transmettre dans le temps les idées qui ont porté à son origine. Aussi, la valeur technique et scientifique que cet ouvrage prend dans le temps, l'expression d'un savoir-faire technologique et structurel caractérisant une certaine période dont il est le témoin principal, est à la base de notion de bien culturel. Dans de telles circonstances, on peut donc bien parler tant de patrimoine du paysage à protéger dans son ensemble que de patrimoine architectural à protéger individuellement.

Réfléchissons par exemple sur les innombrables cas liés au patrimoine architectural ou d'ingénierie, c'est-à-dire à toutes ces œuvres qui constituent des exemples remarquables d'un type de construction, et qui doivent être considérés soit comme une partie intégrante d'un paysage, soit comme biens architecturaux individuels qui doivent être préservés pour leurs qualités esthétiques et technologiques, c'est-à-dire pour l'application d'une technique ou d'un matériau particulier ou même pour l'utilisation d'une méthode d'exécution originale et innovante. À partir de ces considérations, et en accordant l'attention à l'objet principal de notre discussion, à savoir le béton armé, on peut dire que de nombreuses œuvres réalisées dans les premières décennies du $\mathrm{XX}^{\mathrm{e}}$ siècle avec cette nouvelle technique de construction (ponts, bâtiments industriels, infrastructures de toutes sortes et bâtiments civils) devraient être considérées comme des œuvres collectives de la technique et de la culture historique du bâti et pour cette raison protégées comme des « œuvres d'art ».

On pense par exemple au cas des centrales hydroélectriques. Elles sont un musée à ciel ouvert de comment la ressource en eau peut être utilisée et transformée en énergie électrique. En observant attentivement toutes les étapes de la production d'électricité dans ces ouvrages architecturaux, c'està-dire de la collecte de l'eau dans les grands bassins de montagne à leur concentration dans les réservoirs de la vallée, des barrages aux canaux d'approvisionnement aux centrales électriques, des cabines pour la transformation de l'énergie aux lignes électriques, nous pouvons bien comprendre la nature spécifique de ce patrimoine technique et historique qui prend, pour ses caractéristiques typiques, des qualités culturelles extraordinaires.

L'environnement autour de ces ouvrages d'art lui-même devient une partie intégrante de ce patrimoine, revenant ainsi à cette idée de paysage culturel que j'ai mentionné plus tôt. Ces œuvres architecturales, en fait, méritent d'être incluses dans la notion de patrimoine culturel à la fois individuellement, comme expression du complexe technique dont elles jouissent, et à la fois en tant que partie intégrante du patrimoine environnemental; à savoir en relation avec l'environnement naturel auquel elles appartiennent qui, dans certains cas, s'est justement caractérisé par la présence de ces ouvrages. Pensons, par exemple, au cas des centrales du Parc d'Adda Nord, en Italie, qui sont toutes ensemble un véritable cas de paysage culturel et de «route industrielle » de l'énergie hydroélectrique.

Une centrale hydroélectrique, en effet, doit être considérée comme la réalisation d'une idée de progrès capable de grands changements et d'un développement social et industriel généralisé. Elle doit également être perçue non seulement comme un lieu de production, mais aussi comme un moyen d'image et de communication. D'après ce qu'on a 
dit, nous comprenons que de nombreuses centrales hydroélectriques ont également eu des caractéristiques formelles et esthétiques de valeur remarquable. En effet, dans l'élaboration des projets, plusieurs sociétés d'électricité sélectionnaient les architectes les plus célèbres pour mettre en évidence leur image à travers un résultat de grande qualité architecturale. La plupart des architectes appelés à cette tâche ont essayé, souvent dans un cadre original et innovant, de réinterpréter les différentes langues et styles architecturaux du moment en les appliquant à ce type d'ouvrage industriel. Les formes de l'Art Nouveau, du classicisme, de l'éclectisme, du rationalisme sont alors habilement utilisées dans ces œuvres architecturales, choisies comme symbole d'avantgarde et de renouvellement culturel.

Cette immense richesse de l'archéologie industrielle liée à l'eau doit être protégée également pour sa valeur architecturale. On pense aux raffinements architecturaux des centrales italiennes de Robbiate, de Taccani, de Esterle, de Campovico, de Turbigo, de Crevola, de Bordogna, pour n'en nommer que quelques-unes, qui fournissent un exemple intéressant d'architecture moderne basée sur les décorations en plâtre et sur les références aux éléments architecturaux du passé tels que les timbales, les fenêtres circulaires ou les fenêtres jumelées; on pense également aux nombreuses centrales qui reflètent l'influence du rationalisme architectural, telles que les centrales Bard et Perrères dans le Val d'Aoste ou la centrale Cedegolo en province de Brescia (Italie), et d'autres encore qui, en adoptant de larges ouvertures verticales, mettent davantage l'accent sur la grille de structure en béton armé. Dans ce dernier cas, alors, elles deviennent aussi l'exemple d'un changement esthétique de la forme architecturale qui dérive de l'adoption encore plus raisonnée du béton armé. Toutefois, en dépit de ces considérations, une grande partie de ce patrimoine industriel est perdu dans le néant.
Dans le choix de la mise en valeur de ces ouvrages architecturaux, et aussi dans la reconnaissance patrimoniale des autres œuvres en béton armé, et en tenant compte que tout ne peut pas être considéré comme un patrimoine à protéger, on se demande alors quels devraient être les « critères essentiels » pour l'évaluation correcte de ces œuvres ; à savoir les critères d'identification de leur valeur monumentale. Comme déjà mentionné, la valeur esthétique, la valeur historique des technologies innovantes et le témoignage d'innovations fonctionnelles, jouent certainement un rôle important dans l'identification de ce qui est considéré « patrimoine culturel ». Référées au sujet du béton armé, ces valeurs deviennent plus vraies que jamais.

Depuis le début du siècle dernier, en effet, le béton armé a été reconnu comme l'une des découvertes les plus importantes de l'ingénierie structurelle, surtout en raison de son caractère révolutionnaire et novateur qui en quelques années conduira à un changement radical de la façon d'entendre l'architecture. Les nombreux avantages de la nouvelle technique ne sont pas suffisants (coût, simplicité et rapidité d'exécution, résistance au feu, "hygiène ») pour expliquer comment le béton armé a réussi à gagner aussi rapidement et efficacement une place privilégiée dans le monde de la construction. Ce qui est certain, c'est qu'en un siècle seulement, des premières applications des inventeurs et expérimentateurs audacieux de la fin $\mathrm{du} \mathrm{XIX}^{\mathrm{e}}$ siècle à la réalisation des premières constructions des architectes du $\mathrm{XX}^{\mathrm{e}}$ siècle, la technologie du béton armé est devenue le leader incontesté d'une nouvelle ère de la construction s'élevant au rang de paradigme absolu de la modernité et du progrès. Avec raison, on pourrait définir cette combinaison de fer et conglomérat, tout à fait ingénieuse, comme l'une des inventions les plus brillantes du siècle passé qui a joué un rôle essentiel dans la deuxième révolution industrielle. À savoir la véritable «pierre philosophale » du $\mathrm{XX}^{\mathrm{e}}$ siècle ${ }^{2}$. 
En outre, sa présence sera fondamentale dans le scénario de reconstruction qui se déterminera dans toute l'Europe après la Deuxième Guerre mondiale, comme bien l'expliquera le ministre des Travaux publics, des Transports et du Tourisme de la France de l'époque, à savoir M. Christian Pineau, qui en 1949 lors de la cérémonie d'ouverture des « Journées du Centenaire » dira :

« [...] Dans cette œuvre de reconstruction, le béton armé a joué un rôle énorme. Nous ne pouvons même pas imaginer ce qu'elle aurait été sans lui, de combien d'années ou de décennies on aurait eu besoin pour accomplir le même travail de reconstruction employant matériaux et techniques constructives traditionnels $[. .] »$.3 .

Sans le béton armé, l'extraordinaire boom économique et constructif qui a caractérisé l'aprèsguerre de la plupart des pays européens n'aurait probablement jamais eu lieu. Ces brèves réflexions sur la valeur historique de la nouvelle technique de construction permettent d'attribuer à certains travaux en béton armé cette valeur monumentale dont nous avons parlé. Du point de vue formel, par contre, le nouveau matériel a permis aux ingénieurs de dépasser les limites des structures lourdes considérées jusque-là comme insurmontables, en particulier en termes de lumière et de hauteur, et d'atteindre les finesses propres des structures métalliques.

Pensons aux nombreux cas offerts par les ouvrages d'ingénierie où le béton a démontré ses performances exceptionnelles. Le défi des grands ponts du XXe siècle n'est qu'un des exemples possibles. Encore une fois, les œuvres liées à l'eau reviennent dans notre discours comme exemple fondamental de la technique et de l'histoire architecturale. Après plusieurs années d'applications modestes, depuis les premières années du $\mathrm{XX}^{\mathrm{e}}$ siècle, le nouveau matériau est finalement utilisé pour la mise en œuvre complète d'importants travaux publics. La construction, en 1911, du Pont Risorgimento à Rome (caractérisé par une lumière de 100 mètres considérée comme impensable à l'époque) n'est que l'un des nombreux cas auxquels on peut faire référence. Conçu par
François Hennebique, c'est une véritable œuvre de l'ingéniosité humaine. Pour ses caractéristiques innovantes, telles que la présence de deux coquilles reliées à des diaphragmes verticaux ou encore l'utilisation de barres d'acier à haute adhérence, ce pont doit être considéré comme un jalon dans l'histoire des infrastructures et à ce titre doit être admiré et conservé, même si en réalité il est encore dépourvu de mentions venants de la «culture » officielle. Les ponts de Hennebique ont été suivis par toute une génération de grands ponts semblables qui doivent être rappelés pour leurs excellentes qualités esthétiques et structurelles. Prenons par exemple en considération les ponts de Robert Maillard, dont certains sont aujourd'hui conservés avec grand soin en Suisse, dans lesquels l'intention d'attribuer la force axiale à une coque mince et l'action de flexion à une plate-forme solide et rigide se traduit par la création de formes architecturales très intéressantes pour l'époque. Plus tard, la technique du béton précontraint de Freyssinet, un autre jalon dans l'histoire des structures, révolutionnera la construction des ponts successifs en béton armé, en déterminant à mon avis de véritables chefs-d'œuvre. Cela étant dit, une question spontanée se pose: dans l'avenir, ne devrait-on pas conserver et admirer comme un monument merveilleux, le Pont de Luzancy sur la Marne de Eugene Freyssinet. Construit en 1945, mais conçu en 1941, en même temps que les autres ponts-frères proches de lui, ce pont doit être considéré à mon avis comme le témoignage le plus spectaculaire d'une nouvelle technique de construction née d'un calcul scientifique et non d'une évolution de la pratique comme cela se passait d'habitude. Pour cette raison, la valeur conceptuelle et scientifique du pont doit être considérée comme «le motif exceptionnel » nécessaire à sa préservation.

La tâche de la communauté scientifique doit être celle de reconnaître et de préserver de telles œuvres comme témoignages précieux d'une époque qui doit être transmis à travers toute la richesse de ses 
innovations technologiques. Cependant, ce résultat souhaitable n'a pas toujours été atteint avec facilité. Prenons le cas de l'Italie. Bien que les différentes chartes de la restauration, auxquelles on se réfère pour la protection et la préservation des biens (voir la Charte de Venise de 1964, la Charte pour la conservation et la restauration des objets d'art et de la culture de 1987, pour ne nommer que celles-ci), indiquent clairement que la notion de monument est appliquée pour chaque époque et zone géographique considérées importantes du point de vue artistique, historique et culturel en général ; il est aussi tout à fait vrai que l'attention est toujours orientée vers des œuvres artistiques et monumentales «anciennes », tandis que l'espace du moderne comme patrimoine en danger est encore tout à déterminer.

En outre, dans le cas des travaux d'infrastructure, industriels et civils, qui ont été une réussite et une fructueuse forme d'expression de l'histoire du béton armé, la conscience de la valeur monumentale, en tant que témoignage culturel et historique du développement technologique, n'est pas perçue au niveau administratif et politique. Ainsi, de nombreuses œuvres de grand intérêt technique, architectural et esthétique, courent le risque de disparaître: on pense par exemple au Pont Camille de Hogues à Châtellerault (Vienne), premier pont en béton armé système Hennebique de la ville, qui a été classé monument historique seulement en 2002 avec pour résultat la restauration d'éléments structuraux; on pense également aux débats qui dans ces années ont caractérisé certains ouvrages de l'architecture contemporaine comme par exemple « les bâtiments de la Marine nationale », conçus par Auguste Perret dans le $15^{\mathrm{e}}$ arrondissement de Paris, actuellement menacés de destruction en raison d'un vaste projet du Ministère de la Défense; ou « la Station sanitaire du port de Marseille» de Fernand Pouillon, menacée elle-même de démolition par la Municipalité. Dans le cas spécifique des bâtiments d'Auguste Perret, la démolition de ces structures mènerait à la disparition d'une partie de l'héritage de cet architecte qui a été très important dans le domaine du béton armé, sans compter que cet héritage a été déjà mutilé par la démolition du Garage en rue Ponthieu et des Ateliers Esders. Les bâtiments de la Marine nationale doivent donc être protégés. La même réflexion a été faite, en ces années, par la Docomomo France qui avec une pétition intitulée Sauvons les bâtiments de la Marine Nationale / Paris $15^{e}$. Non à la destruction d'une cuvre majeure des frères Perret, a voulu faire remarquer l'importance de la sauvegarde de ces ouvrages en tant que patrimoine du $\mathrm{XX}^{\mathrm{e}}$ siècle. Ainsi est expliquée la volonté de préservation de ces bâtiments dans la pétition susmentionnée :

«[...] Le dossier d'archives (l'un des plus riches $\mathrm{du}$ fonds Perret) témoigne de l'effervescence qui a accompagné la conception du projet. On peut, grâce à 2000 documents conservés, suivre les recherches qui ont abouti à cette œuvre savante. Dans Le langage de l'architecture classique, John Summerson compare la Marine nationale à l'Opéra de Paris : "Le bâtiment est entièrement en béton armé et dépourvu de tout ornement. Mais il est pensé en termes d'ordres". "Il y a presque autant de relief et de variété, de rythme, dans ce bâtiment, que dans l'Opéra. Simplement, il n'y a ni moulure, ni sculpture". Peter Collins souligne la maitrise de cet ensemble. Le soin accordé aux proportions, le jeu de l'ombre et de la lumière, la composition des bétons révèlent "quelque chose de plus profond que l'intelligente amélioration des éléments architectoniques essentiels. L'architecture industrielle est portée ici au degré le plus élevé de l'art". Seul le bâtiment administratif est inscrit à l'inventaire. Les autres constructions vont disparaître. Démanteler un tel patrimoine pour n'en conserver que la partie administrative est inacceptable. Ce serait perdre les qualités d'harmonisation qui ont porté cette "architecture industrielle" à ce degré de dignité qu'ont su percevoir Summerson et Collins. Ce serait discréditer définitivement la politique de protection du patrimoine du XXème siècle engagée par le Ministère de la Culture [...] »4.

Cela dit, nous devons aussi reconnaître que tous les ouvrages en béton armé ne doivent pas être forcément considérés comme patrimoine à 
protéger. De plus, il ne serait pas souhaitable qu'il y ait une muséification de la totalité des biens du XXe siècle. La question de ce qu'il faut protéger devient alors centrale. Afin de répondre à cette problématique, de nombreuses archives d'ouvrages architecturaux, nées de recensements et catalogages, sont en train d'être diffusées. Celles-ci peuvent aider sur le choix de ce qu'il faut transmettre aux générations futures; un choix fait pas seulement en fonction des critères architecturaux, ou des valeurs liées à la figure professionnelle de l'auteur de l'œuvre en question, plus ou moins appréciée par la critique, mais aussi sur la base de minutieuses et précises réflexions critiques.

Ainsi, par exemple, pour faire partie du patrimoine de l'ingénierie, les travaux en question doivent répondre à des critères spécifiques, tels que « avoir une importante signification dans l'histoire de l'ingénierie, être associé à un événement de l'histoire de l'ingénierie, ou avoir été réalisée par une personnalité illustre de l'ingénierie mondiale ou par un groupe de personnes, ou encore constituer un témoignage remarquable d'une technique, d'un matériau ou d'un type de construction qui a contribué au développement d'une zone déterminée, etc. »5. Si on analyse la liste complète de ces critères, on constate qu'ils sont essentiellement liés à la notion d'innovation structurelle. Toutefois, dans l'évaluation de la valeur monumentale de ces constructions, une égale importance doit être accordée à la notion de valeur artistique et esthétique que telles œuvres possèdent.

On a beaucoup discuté sur la valeur esthétique des œuvres de l'architecture moderne, et en particulier des ouvrages en béton armé, pour atteindre à un compromis équitable autour des années 1960, quand le béton a finalement gagné la compétition sur la pierre grâce à la beauté de sa surface absorbante, ainsi qu'à sa texture et sa couleur qui ont été préférées à celles du marbre et du granit. De ce fait, de plus en plus d'architectes commencèrent à choisir le béton pour les surfaces exposées de leurs œuvres. Considérons, par exemple, Carlo Scarpa, maître des plus sophistiqués verres de Murano, des ouvrages dans la blanche pierre d'Istrie à Venise, ou du rouge marbre de Vérone, qui a été conquis par le béton armé de manière si forte qu'il a formé la plupart de ses chefs-d'œuvre suivants avec cette nouvelle technique constructive. En outre, bien avant cette époque, à savoir dans les années 1930, le béton a été considéré comme le matériau idéal de l'expressionnisme en raison de sa nature plastique et sa capacité à être moulé dans n'importe quelle forme. Le rationalisme trouvera dans la combinaison de cadres, plaques et murs en béton armé, la grille idéale pour son espace architectural cartésien. Cette valeur formelle et esthétique du nouveau matériel a été recherchée et obtenue par de nombreux architectes du XXe siècle (Musmeci, Wright, Nervi) qui habilement ont exploité la capacité du béton armé de s'adonner à l'imagination et à l'audace des concepteurs. Ce qu'on vient de dire trouve confirmation dans les paroles de Pier Luigi Nervi, qui affirmait avec beaucoup de conviction :

«[...] la capacité de créer une pierre artificielle de forme quelconque, supérieure à la naturelle, car elle est capables de résister à la tension, a un je-ne-sais-quoi de magique [...]. Sa nature, malléable avant et après monolithique, est de contrôle difficile, mais elle s'offre, à ceux qui savent la gouverner, comme un outil exceptionnel pour inventer des formes sans précédents, des nouvelles images et des nouveaux espaces pour l'architecture $[\ldots] »^{6}$.

Le succès du béton était tel que même les spécialistes les plus conservateurs ont été attirés par le miracle apparent de ce nouveau matériau, considéré comme plus durable que la brique traditionnelle, et moins vulnérable que la pierre aux attaques de l'environnement. La réalité cependant fut différente. Dans les années 1960, de nombreuses structures en béton du début du $\mathrm{XX}^{\mathrm{e}}$ siècle arrivèrent à l'âge de cinquante ans et l'illusion de l'éternité a été détruite par les premiers effets de la dégradation, capable de créer des sérieux problèmes à la conservation de ces chefs-d'œuvre du passé. 
Cette nouvelle problématique a incité à réfléchir sur l'importance de la préservation de ces œuvres, considérées comme véritables exemples de l'architecture moderne.

À ce stade, avant de passer à la discussion du concept de sauvegarde des œuvres modernes en béton armé, il convient à mon avis de préciser le sens qu'ici on veut donner au terme moderne, car c'est de lui que part le concept de protection. Le moderne auquel on se réfère dans ce cas est également associé à trois sens interdépendants les uns des autres: le sens de la modernisation, liée aux processus de développement scientifique et technologique qui ont caractérisé le $\mathrm{XX}^{\mathrm{e}}$ siècle; le sens de la modernité, référée aux changements sociaux et culturels résultants de la modernisation; et le sens du modernisme, entendu comme représentation esthétique de la modernité. Le patrimoine d'ingénierie et architectural du $\mathrm{XX}^{\mathrm{e}}$ siècle, à mon avis, a toutes ces qualités, ayant été le principal moyen de diffusion de ces nouvelles théories.

Cependant, le manque d'une sensibilité culturelle adéquate des organes de compétences, et la négligence d'une partie de la communauté, a conduit au fil du temps à une obsolescence constructive et à une détérioration générale des architectures en question. Depuis les années 1960, années au cours desquelles la dégradation de certains de ces travaux en béton a commencé à être perçue, le risque de perdre cette architecture qui, plus que de simples bâtiments, sont de véritables affiches des nouvelles idéologies, a conduit à réfléchir sérieusement sur la question de la préservation de ce patrimoine. Cette question a été abordée en première analyse en référence au seul patrimoine architectural réalisé selon les enseignements du Mouvement Moderne. Au vu de l'énorme écho que ses principes révolutionnaires ont eu depuis leur naissance, ils ont été étendus à d'autres travaux d'architecture du $\mathrm{XX}^{\mathrm{e}}$ siècle dignes de mention. Comment pouvons-nous oublier, par exemple, l'histoire du sauvetage de Villa Savoye de
Le Corbusier, qui a vu l'auteur même s'opposer à la démolition du bâtiment en mobilisant l'intérêt des critiques et des intellectuels de la période afin de faire reconnaître son œuvre comme un manifeste de l'esthétique moderne de l'architecture. À la suite de cet effort collectif, en 1964, on décidera de classer l'œuvre comme Monument Historique National, déterminant un événement tout à fait exceptionnel pour la France de l'époque.

L'importance de la reconnaissance par une communauté des valeurs historiques et culturelles d'un bien, que ce soit un bâtiment ou un pont ou tout autre ouvrage du génie de l'homme, devient donc la première étape pour sa conservation. Ce qui précède est clairement démontré par d'autres vicissitudes liées, une fois de plus, aux architectures de Le Corbusier. C'est le cas des cinq Unités d'habitation réalisées entre le début des années 1950 et la fin des années 1960 à Marseille, Rezé, Berlin, Briey et Firminy. Toutes ont été conçues pour accueillir un grand nombre de petits logements pour les classes ouvrières qui, au contraire, n'aimeront pas beaucoup l'idée de ces villages populaires, et les abandonneront dans un court laps de temps. Paradoxalement, la sauvegarde de ces architectures «socialistes» a eu lieu quelques années plus tard grâce aux classes supérieures, qui ont redécouvert le sens ancien du projet en les faisant coïncider avec leurs propres besoins, et, surtout, ont compris l'importance de posséder et de vivre dans un morceau d'histoire de l'architecture. Cette prise de conscience a fait que dans chaque habitation est née une communauté, parfois structurée en forme de véritable organisation, capable de promouvoir la restauration des bâtiments, d'obtenir le lien de protection par les autorités compétentes et de continuer à promouvoir la diffusion et la maintenance de ces bâtiments7. La préservation des œuvres de Le Corbusier, qui a contribué à faire devenir Firminy-vert patrimoine de l'Unesco, est à prendre comme point de référence, et non pas seulement comme expression d'un acte culturel, mais aussi comme témoignage 
d'une opération qui a donné au bien en question une valeur économique considérable. Cela est démontré tant par la grande masse de visiteurs qui chaque année se rendent à ces endroits que par l'augmentation significative des locations et des ventes. Cette préservation doit également être perçue comme un exemple d'engagement civil de toute une communauté, qui toute seule a réussi dans sa tentative de sauvegarde, soulignant l'importance de la supervision d'un bien par une société solidaire et participative.

Cela dit, on peut à cet égard se demander quelle est réellement l'attention de la communauté scientifique internationale par rapport à ce sujet? L'augmentation rapide d'intérêt qui a eu lieu dans les dernières années du XXe siècle est confirmée par l'augmentation de séminaires et conférences organisés par plusieurs associations de spécialistes. Parmi les plus actives il faut se souvenir de Do.Co.Mo.Mo (Documentation and Conservation of buildings, sites, and neighbourhood of the Modern Movement), déjà mentionnée, qui a été fondée en 1989 à l'Université Technique d'Eindhoven afin de contribuer à l'amélioration et à la préservation de l'architecture moderne ${ }^{8}$. La particularité de cette association est d'agir comme un lieu de débat où l'on peut discuter de la préservation du patrimoine $\mathrm{du} \mathrm{XX}^{\mathrm{e}}$ siècle, époque passionnante et unique dans laquelle l'architecture, l'urbanisme et l'aménagement paysager ont subi une transformation importante grâce à la volonté de rompre radicalement avec les théories du passé. Son objectif principal est donc de mettre l'accent sur les valeurs d'innovation du mouvement moderne, qui impliquent à la fois la sphère technique, esthétique et sociale. Grâce à un catalogage des bâtiments et des sites, réalisé selon des critères spécifiques, l'objectif que Docomomo vise à atteindre est la création de contraintes précises de sauvegarde sur ces ouvrages, étape préliminaire et nécessaire pour obtenir une protection ultérieure.
Il faut se rappeler que d'autres organisations nationales et internationales travaillent pour la protection $\mathrm{du}$ patrimoine architectural du $\mathrm{XX}^{\mathrm{e}}$ siècle, en élargissant le regard à toutes les autres œuvres de cette période, y compris celles industrielles. C'est le cas de l'ECCE (European Council of Civil Engineers), de l'ICE (Institution of Civil Engineers), de l'ASCE (American Society of Civil Engineers), du CILAC (Comité d'information et de liaison pour l'archéologie, l'étude et la mise en valeur du patrimoine industriel), de l'AIPAI (Associazione Italiana per il Patrimonio Archeologico Industriale), et au niveau International du TICCIH (The International Commitee for the Conservation of Industrial Heritage) et surtout de l'ICOMOS, qui a récemment porté son attention vers ce patrimoine à travers la création de deux comités qui s'y intéressent de façon directe, l'ISC2OC, ou indirecte, l'ISCARSAH. Le premier s'occupe de l'identification, de la conservation et de la protection des œuvres du siècle dernier ; le second favorise la coopération de la communauté scientifique internationale, pour ce qui concerne la connaissance des aspects structurels de tous les bâtiments protégés, établissant des lignes directrices pour procéder correctement à leur préservation et diffusant dans les différents pays les connaissances acquises 9 D'autre part, la connaissance approfondie des techniques de construction des bâtiments apparaît être l'outil essentiel et principal pour leur bonne conservation.

À cette phase de croissance d'intérêt pour l'architecture contemporaine, il devait toutefois correspondre une législation appropriée. La sauvegarde du patrimoine de l'architecture moderne en Europe a été soutenue par des instruments juridiques qui ont essayé de combler le vide juridique qui existait sur le sujet. L'expérience française est, peut-être, la plus exemplaire à la fois pour la précocité avec laquelle elle est apparue et pour l'ampleur de son action. Déjà en 1957 ont été initié par l'inspecteur général Chauvel, «les premières campagnes cognitives thématiques de la 
modernité ", sans toutefois aucune distinction normative entre le patrimoine architectural en général et celui de l'architecture contemporaine. Par la suite, en 1961, cet inconvénient fut surmonté avec la réalisation de l'Inventaire supplémentaire, dans lequel furent inscrits les ouvrages de l'architecture contemporaine les plus remarquables. Dignes de mention, pour l'engagement adopté en termes de sauvegarde du patrimoine moderne, citons aussi l'Allemagne et l'Angleterre: la première pour sa contribution à la protection de certains cas emblématiques, tels que les divers sièges de l'école du Bauhaus, et la seconde pour ses efforts internationaux promus par le Twentieth Century Society et le National Trust, organisme gouvernemental chargé du patrimoine historique anglais.

Par contre, en analysant plus en détail le cas italien, nous pouvons avoir une idée de comment la reconnaissance de la valeur monumentale d'une œuvre contemporaine par un État est une chose longue et difficile. Lorsqu'au milieu des années 1960, la commission parlementaire «Franceschini » recueille une quantité importante de matériel afin de faire le point de la situation sur l'état du patrimoine culturel national et sur sa gestion, le patrimoine moderne est analysé seulement en termes de compatibilité environnementale, paysagiste et urbanistique. Dans les mêmes actes de la Commission, on trouve également une allusion aux débats qui à l'époque critiquaient fortement, pour leur incompatibilité environnementale, les mêmes bâtiments qui aujourd'hui sont considérés comme des chefsd'œuvre (pensons à la «Torre Velasca » à Milan, à cette période-là considérée comme un exemple de dégradation du centre historique de la ville et du paysage ${ }^{10}$. Dans les anciens instruments normatifs qui s'occupaient du patrimoine architectural n'apparaît aucune disposition de protection spéciale pour l'architecture contemporaine. Ce n'est qu'à la fin des années 1990, lorsqu'il y a eu la naissance d'une multitude de règles pour renouveler l'organisation de la protection et de la sauvegarde des biens, qu'on a commencé à prêter attention aux témoignages de l'architecture moderne. En 1998, le nouveau Ministère des biens et des activités culturelles est créé11 et, parmi les différentes directions générales conçues, est réalisée une direction spécifique pour l'art et l'architecture contemporaine : la DARC (Direction générale pour l'Architecture et pour l'Art contemporaine) ${ }^{12}$. Son rôle sera important pour une plus grande prise de conscience du sujet ${ }^{13}$.

Durant ces dernières années, l'engagement de chaque pays a été remarquable. En Europe, à partir de 1991, ont été préparés certains principes pour la préservation et la valorisation des œuvres du $\mathrm{XX}^{\mathrm{e}}$ siècle. Nous nous souvenons par exemple des «Recommandations $\mathrm{n}^{\circ} 13$ du Comité des Ministres des États membres " intitulées The protection of the twentieth-century architectural heritage. Malgré cela, il y a encore de nombreuses constructions en béton armé, comme des ponts, bâtiments industriels, réservoirs, silos, quais, tours, dont parfois on n'a aucune connaissance, qui luttent pour être reconnus comme patrimoine de valeur inestimable. Ce qu'on espère est un travail de documentation sérieux et rigoureux qui permette d'avancer, à partir des différents États, une hypothèse de protection et de mise en valeur de ces biens, dans le respect de ce que l'héritage du passé nous a donné et dans la responsabilité de ce qui, à son tour, doit être transmis aux générations futures en vue d'une continuation de la mémoire historique.

En revanche, comme l'a bien expliqué Simmel dans son essai sociologique sur la ruine ${ }^{14}$, la capacité de l'architecture est de donner le souvenir du passé dans la matière construite, c'est-à-dire de relancer et de compléter le passé dans le présent. La mémoire historique, le souvenir, trouve en fait son intermédiaire privilégié véritablement dans les œuvres d'architecture, grâce à une osmose continue de significations et d'expériences entre passé, présent et futur. Tout cela doit être pris en considération quand on se rapporte à un bien à 
protéger. Alors, par rapport à cela, les paroles du fameux livre de John Ruskin, The seven Lamps of Architecture, deviennent très claires, lorsque, en se référant à l'architecture, il dit :

«[...] nous pouvons vivre sans elle, nous pouvons même adorer sans elle, mais nous ne nous pouvons pas nous souvenir sans elle $[\ldots] » 15$.

1 RUSKIN John, " Presentazione ", Le sette lampade dell'architettura, trad. it. a cura di Renzo M. Pivetti. Milano, Jaca Book, «Presentazione, 1981, p.19. (Traduit de l'italien par Mariangela Licordari).

2 Pour plus d'informations sur le sujet cf. DELHUMEAU Gwenael, L'invention du béton armé : Hennebique, 1890-1914. Paris, Norma, 1999; IORI Tullia, Il cemento armato in Italia dalle origini alla seconda guerra mondiale. Edilstampa, Roma 2001; SIMONNET Cyrille, Le béton. Histoire d'un matériau. Paris, Éd. Parenthèses, 2005.

3 MEZZINA Mauro, UVA Giuseppina, Degrado e riabilitazione strutturale delle architetture in cemento armato. Actes du $15^{\circ}$ Congrès C. T. E. Bari, 4-5-6 novembre 2004, p. 2.

4 Pétition Docomomo France Sauvons les bâtiments de la Marine Nationale / Paris $15^{e}$. Non à la destruction d'une ouvre majeure des frères Perret, p. 1.

5 «Le patrimoine d'ingénierie : 150 ans d'innovations structurales à Bruxelles », dans L'aménagement du territoire en question, actes de la journée d'études du 7 Juin 2011, pp. 8-9.

6 SICIGNANO, Architetture in cemento armato. Napoli, Clean Edizioni, 2007, p. 154 (traduit de l'italien par Mariangela Licordari).

7 Dans l'Unité d'habitation de Rezé, appelée Maison Radieuse, par exemple, est née l'AHMR (Association des Habitants de la Maison Radieuse) qui organise la vie collective et les activités de loisirs du village vertical; tandis que la préservation de la Cité Radieuse de Briey-en-Foret a eu lieu grâce à la création de l'association La Premier Rue, constituée par des architectes, intellectuels et artistes.

8 En 1995, cette association a trouvé son siège italien à l'Université de Rome Tor Vergata.
9 Cf. La carte ICOMOS sur les « Principes pour l'analyse, la conservation et la restauration structurale du patrimoine architectural », XIV Assemblée Générale de l'Icomos, Victoria Falls (Zimbabwe), octobre 2003; sur la même argumentation voir aussi : "The protection of the twentieth-century architectural heritage ", dans Recommendation n. 13 of the Committee of Ministers to member States, septembre 1991.

10 Cf. «Actes de la Commission Franceschini, 1964-66 », dans Per la salvezza dei beni culturali in Italia. Atti e documenti della Commissione d'indagine per la tutela e la valorizzazione del patrimonio storico, archeologico, artistico e del paesaggio. Roma, Ed. Colombo, 1967.

11 Cf. D. Lgs. 20 ottobre 1998, n. 368, Istituzione del Ministero per $i$ Beni e le Attività Culturali, a norma dell'art. 11 della legge n. 59 del 15 marzo 1997

12 Par la suite, avec l'entrée en vigueur du D.P.R. n. 233 de 2007 (art. 7, comma 1e 2), la DARC deviendra DPARC, Direzione Generale per la qualità e la tutela del Paesaggio, l'Architettura e l'Arte Contemporanee, afin d'étendre les pouvoirs de la Direction aussi sur le paysage.

13 Actuellement, pour soumettre les bâtiments modernes aux pratiques de protection on se réfère au Code Urbani (D. Lgs. $n$ 42 del 22 gennaio 2004, Codice dei Beni Culturali e del Paesaggio) pour la protection de ces biens qui ont plus de cinquante ans et dont l'auteur n'est plus en vie, ou à la loi sur le droit d'auteur (Legge 22 aprile 1941, n. 633, Protezione del diritto d'autore e di altri diritti connessi al suo esercizio) dans le cas dont l'œuvre n'a pas encore cinquante ans et son auteur est vivant. Dans ce dernier cas c'est l'auteur même à demander que à son œuvre soit reconnue la valeur culturelle, accordée seulement après vérification préalable du Ministère.

14 SIMMEL Georg, "Die Ruine », Philosophische Kultur. Gesammlte Essays, Gesammtausgabe, tome 14, Francfort, Suhrkamp, 1996.

15 RUSKIN, Le sette lampade dell'architettura, cap.VI, trad. it. a cura di Renzo M. Pivetti, Milano, Jaca Book, 1981, p. 209. (Traduit de l'italien par Mariangela Licordari). 


\section{Bibliographie}

«Actes de la Commission Franceschini, 1964-66 ", dans Per la salvezza dei beni culturali in Italia. Atti e documenti della Commissione d'indagine per la tutela e la valorizzazione del patrimonio storico, archeologico, artistico e del paesaggio. Roma, Ed. Colombo, 1967.

AA.VV. Carta Icomos - Principes pour l'analyse, la conservation et la restauration structurale $d u$ patrimoine architectural, XIVe Assemblée Générale de l'ICOMOS, Victoria Falls (Zimbabwe), 2003.

CASCIATO Maurizio, MORNATI Stefania, ET PORETTI Sergio, Architettura moderna in Italia: documentazione e conservazione, primo convegno nazionale Do.co.mo.mo Italia. Roma, Edilstampa srl., 2000.

DELHUMEAU Gwenael, L'invention du béton armé: Hennebique, 1890-1914. Paris, Norma, 1999.

IORI Tullia, Il cemento armato in Italia dalle origini alla seconda guerra mondiale. Edilstampa, Roma, 2001.

Le patrimoine d'ingénierie: 150 ans d'innovations structurales à Bruxelles, dans «L'aménagement du territoire en question», actes de la journée d'études du 7 Juin, 2011.

MEZZINA Mauro, et UVA Giuseppina, Degrado $e$ riabilitazione strutturale delle architetture in cemento armato. Actes du $15^{\circ}$ Congrès C.T.E. Bari, 4-5-6 novembre, 2004.

RUSKIN John, Le sette lampade dell'architettura, trad. it. a cura di R.M. Pivetti, Milano, Jaca Book, 1981.

RIEGL Alois, Le culte moderne des monuments: son essence et sa genèse. Paris, Editions du Seuil, Collection Espacements, 1984.

SIMMONNET Cyrille, Le béton. Histoire d'un matériau, Éd. Parenthèses, 2005.

SICIGNANO Enrico, Architetture in cemento armato. Napoli, Clean Edizioni, 2007.
"The protection of the twentieth-century architectural heritage » dans Recommendation $n$. 13 of the Committee of Ministers to member States, 9 September 1991, 1991.

ZEVI Bruno, Storia dell'Architettura moderna. Torino, Einaudi Editore, 1990. 